\title{
Implementación de un programa de rehabilitación cardiaca (Fase II) en el Hospital Max Peralta
}

\author{
Marlene Roselló-Araya', Sonia Guzmán-Padilla²
}

\begin{abstract}
Justificación y objetivo: Dentro de la prevención secundaria de las enfermedades cardiovasculares los programas de rehabilitación cardiaca (PRC) juegan un papel muy importante. Se presentan en este artículo los resultados del primer grupo de pacientes participantes del PRC desarrollado en el Hospital Max Peralta de Cartago.
\end{abstract}

Métodos: Se implementó un PRC para pacientes que habían sufrido un evento cardiaco, de enero a diciembre de 1999, el cual consistió de sesiones educativas en forma grupal, tres horas una vez por semana durante tres meses. Al inicio y final se evaluaron el perfil lipídico, la antropometría, la dieta y la calidad de vida.

Resultados: Participaron 15 pacientes, con una edad promedio de 59 años, más del $70 \%$ presentaron factores de riesgo de enfermedades cardiovasculares. El 59\% del grupo presentó obesidad o sobrepeso. Al final del programa, el perfil lipídico mejoró principalmente los valores del colesterol y las LDL, la dieta fue nutricionalmente más balanceada, y la frecuencia en el consumo de frutas, vegetales y lácteos aumentó. Por el contrario la frecuencia de consumo de carnes, dulces, grasas y cereales disminuyó. La calidad de vida mejoró del inicio $(96 \pm 22,9)$ al final $(89,7 \pm 27,3)$.

Conclusión: Con el establecimiento de PRC se observa una clara mejoría del paciente post evento coronario en los aspectos físicos y emocionales. Sin embargo, estos programas son difíciles de implementar en los centros hospitalarios.

Palabras claves: Programa de rehabilitación cardiaca, evento cardiaco, lípidos, educación, nutrición, dieta, calidad de vida.

Recibido: 05 de agosto, 2003

Aceptado: 22 de enero, 2003

En Costa Rica en los últimos años, las enfermedades del aparato circulatorio se han convertido en un problema de salud pública. Para 1998 la tasa de mortalidad por enfermedades cardiovasculares fue de 11,2 por 10000 habitantes. El primer lugar por causa específica lo ocupa la enfermedad isquémica cardiaca con una tasa de 5,4 por 10000 habitantes, donde el infarto de miocardio ocupa cerca del $50 \%$ de las muertes en este grupo ${ }^{1}$.

Dentro de la prevención secundaria de las enfermedades cardiovasculares la rehabilitación cardiaca (RC) juega un papel muy importante. En sus inicios se basó en programas de ejercicio físico, las que surgieron después de que se aceptó la movilización de los enfermos con infarto de miocardio ${ }^{2}$; sin embargo se requiere de un tratamiento o intervención integral que es parte esencial de la atención a la que deben tener

\footnotetext{
Abreviaturas: PRC,IAM, IMC, cc/ca,CV, OMS

Instituto Costarricense de Investigación y Enseñanza en Nutrición y Salud (Inciensa).

Correspondencia: Marlene Roselló A. Apdo. 4, Tres Ríos, Cartago, Costa Rica. Correo electrónico:mrosello@inciensa.sa.cr.
}

acceso todos los pacientes cardiópatas. Sus objetivos son mejorar la capacidad funcional, aliviar o disminuir los síntomas y permitir al paciente volver a desempeñar un papel satisfactorio en la sociedad ${ }^{3}$.

Los programas de rehabilitación cardiaca (PRC) como estrategia preventiva, tienen efectos positivos en el desarrollo psicosocial y físico del paciente, tales como la reducción de los factores de riesgo, la disminución de éstos por hospitalización y además reducción de la tasa de morbilidad y mortalidad en pacientes de infarto agudo del miocardio (IAM) u otros ${ }^{3}$.

Se ha determinado que la rehabilitación cardiaca reduce en cerca de un $20 \%$ la muerte cardiovascular y en $37 \%$ la muerte súbita, después del primer año de ocurrido el evento ${ }^{4}$. Hedback y colaboradores encontraron que cinco años después de que un grupo de pacientes infartados recibió rehabilitación cardiaca, éstos presentaron disminución significativa en el riesgo de infarto de miocardio no fatal, menos eventos cardíacos y notaron un aumento en el número de individuos que retornaron a sus trabajos ${ }^{5}$. 
Además, se han observado mejoras en estos pacientes, en los niveles de triglicéridos, HDL- colesterol, LDL-colesterol, tolerancia a la glucosa, peso corporal y capacidad de ejercicio ${ }^{6}$. Sin embargo, existen numerosos aspectos no resueltos que influyen en la recuperación, evolución y calidad de vida (CV) del paciente que ha sufrido un infarto agudo del miocardio. La valoración de la calidad de vida persigue como finalidad última conciliar los elementos objetivos y subjetivos incluidos en los conceptos de salud y enfermedad ${ }^{7}$.

Conociendo que en Costa Rica las únicas experiencias de PRC se desarrollan en el Centro Nacional de Rehabilitación (CENARE) y en el Hospital San Vicente de Paúl, Heredia, surgió la necesidad de implementar estos programas en otros centros hospitalarios.

Como a los pacientes de las provincias, se les dificulta el acceso al CENARE, y ante la elevada incidencia de eventos coronarios en los hospitales regionales como el Hospital Max Peralta de Cartago, se seleccionó éste para implementar un PRC como plan piloto. Además, este centro cuenta con recurso humano interdisciplinario capacitado y motivado en este tipo de programas.

Los objetivos de este estudio fueron "Implementar el PRC CENARE-INCIENSA en el Hospital Max Peralta de Cartago,con el fin de transferirlo a otros centros hospitalarios del país y por medio de éste modificar los hábitos de alimentación, de actividad física, el perfil lipídico y antropométrico y estilo de vida de los pacientes que sufrieron un evento coronario. Se presentan en este artículo los resultados de bioquímica, nutrición y calidad de vida, del primer grupo de pacientes participantes del PRC desarrollado en el Hospital Max Peralta de Cartago.

\section{Metodología}

El equipo interdisciplinario se formó con los profesionales (nutricionista, cardiólogo, microbiólogo, trabajadora social y fisioterapista) del Hospital Max Peralta de Cartago interesados en participar en el PRC, a los cuales se les dio a conocer la metodología del mismo.

Para este estudio se identificaron los egresos hospitalarios por IAM entre enero y diciembre del 1999, (59 casos), para lo cual se revisaron los expedientes clínicos. Se incluyeron los hombres y las mujeres menores de 76 años de edad, se excluyeron aquellas personas que presentaran alguna enfermedad no controlada y que les imposibilitaría desarrollar el programa, se obtuvo además, el número telefónico y dirección de su residencia para contactarlos. Del total, 44 pacientes quedaron fuera del estudio por las siguientes razones: fue imposible contactar al paciente porque el número telefónico o dirección reportado no correspondía, no aceptaron participar y por muerte, por lo que se trabajó únicamente con 15 personas, las cuales fueron citadas para explicarles personalmente el estudio y obtener entonces el consentimiento informado. Cabe mencionar que cada paciente asistió al programa acompañado de uno o más familiares.
El PRC se planteó para ser desarrollado en un período de tres meses en la fase II, debido a que la literatura afirma que este es el tiempo que requiere el paciente desde el momento de la alta hospitalaria hasta la reincorporación del enfermo a su vida normal para elevar la capacidad funcional ${ }^{8,9}$.

Los pacientes asistían tres horas, una vez a la semana; durante la primera hora se realizaba la terapia física con ejercicios de bajo impacto (extensión de cuello, espalda, cadera, cintura, pantorrilla, cuadríceps,cruzar el codo, caminar, subir la rodilla, flexión de rodilla, extensión de piernas, dar pasos hacia atrás y hacia adelante en forma alterna) impartido por la terapista física, aunque se contempló en el estudio, no fue posible realizar pruebas de esfuerzo para medir la capacidad aeróbica. Durante las dos horas siguientes se desarrollaban las sesiones educativas en forma grupal y participativas, con los temas de mayor interés que fueron identificados en los grupos focales realizados previamente con los mismos participantes. Los temas tratados fueron:

1. Principios de dieta saludable

2. Composición de los alimentos, hábitos y formas de cocinar

3. Elaboración de menús

4. Los lípidos y su relación con la enfermedad cardiovascular

5. Que es el infarto?, cuáles son factores de riesgo cardiovascular?, medicamentos en cardiología

6. Autoestima, stress, depresión e importancia del apoyo familiar

7. Importancia del ejercicio físico

8. Aprendiendo a leer etiquetas nutricionales

9. Actividad sexual después del infarto

10. Comiendo fuera de casa

Al inicio del PRC se evalúo en los participantes: el perfil de lípidos sanguíneos, la antropometría (peso, talla, índice de masa corporal (IMC) cintura, cadera), la relación cintura/cadera (cc/ca). En los hombres debe ser < 1,0 y en las mujeres $<0,85$ para evitar riesgo cardiovascular, también se evaluaron los aspectos clínicos (presencia de factores de riesgo), la calidad de vida, la dieta (composición y patrón alimentario), para lo cual se utilizó el registro de consumo de alimentos de tres días y la frecuencia de consumo de alimentos. Para medir indirectamente el impacto de las sesiones educativas, al final del programa los participantes fueron evaluados.

El perfil lipídico se realizó con un equipo marca Targa, y dos reactivos de la casa Wiener, se utilizó el método enzimático AA para el colesterol, monofase AA para las HDL, y color gto/Pap AA para los triglicéridos. Las LDL se calcularon con la fórmula de Friedwald (Comunicación personal por el MQC del laboratorio clínico del Hospital Max Peralta, Cartago) 
Para la calidad de vida se utilizó el cuestionario elaborado y validado en pacientes post-infarto de Velasco et al. ${ }^{10} \mathrm{El}$ cual consta de 44 ítemes divididos en ocho áreas: salud, sueño y descanso, comportamiento emocional, proyectos de futuro, movilidad, relaciones sociales, comportamiento de alerta, comunicación tiempo de ocio y trabajo. Cada ítem vale de 1 a 5 puntos en la escala de Likert La puntación máxima (220 puntos) es para pacientes que refieren una mala $\mathrm{CV}$, mientras que la puntuación mínima (44 puntos) corresponde a los que refieren una buena $\mathrm{CV}$. Los aspectos clínicos, los factores de riesgo, el tiempo de evolución y los medicamentos se obtuvieron por medio de la entrevista directa al paciente.

Se diseñó una base de datos en Epi-info, el análisis se realizó en el mismo programa, utilizando estadística descriptiva y tablas de contingencia.

\section{Resultados}

Participaron 15 pacientes (60\% mujeres) que habían sufrido un infarto agudo de miocardio, la edad promedio del grupo

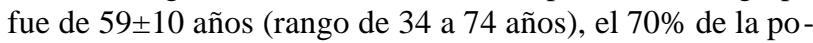
blación presentó más de dos factores de riesgo asociados a las enfermedades cardiovasculares (61,1\% hipertensión, 38,9\% diabetes, 66,7\% dislipidemias). El 59\% de los participantes presentó un IMC que indicaba sobrepeso u obesidad (> 25 $\mathrm{Kg} / \mathrm{m}^{2}$ ). La relación cc/ca mostró una distribución que no implicaba riesgo coronario para los hombres (promedio 0,88), ni para las mujeres (promedio 0,80), al final de la intervención el cambio en estos indicadores no fue estadísticamente significativo.

El perfil lipídico inicial experimentó una disminución particularmente en el colesterol y el LDL, por el contrario los triglicéridos aumentaron, estos cambios fueron estadísticamente significativos (cuadro 1).

La composición nutricional de la dieta determinó que estas personas, al ingresar al PRC ya habían realizado modificaciones en el aporte calórico de su dieta; sin embargo, al final del

\begin{tabular}{|c|c|c|c|}
\hline \multicolumn{4}{|c|}{$\begin{array}{c}\text { Cuadro } 1 \\
\text { Características antropométricas y } \\
\text { bioquímicas de los pacientes } \\
\text { Programa de Rehabilitación Cardiaca } \\
\text { Hospital Max Peralta, } 2000\end{array}$} \\
\hline Variable & $\begin{array}{l}\text { Inicio } \\
\mathrm{n}=15\end{array}$ & $\begin{array}{l}\text { Final } \\
n=15\end{array}$ & $\mathrm{p}$ \\
\hline $\mathrm{IMC}^{1}$ & $27,4 \pm 5,1$ & $26,9 \pm 4,9$ & NS \\
\hline $\mathrm{cc} / \mathrm{ca}^{2}$ & $0,89 \pm 0,04$ & $0,87 \pm 0,05$ & NS \\
\hline Colesterol total $(\mathrm{mg} / \mathrm{dl})$ & $234 \pm 58$ & $211 \pm 36$ & 0,00 \\
\hline Triglicéridos (mg/dl) & $172 \pm 56$ & $197 \pm 75$ & 0,00 \\
\hline HDL-col (mg/dl) & $49 \pm 17$ & $44 \pm 11$ & 0,00 \\
\hline LDL-col (mg/dl) & $140 \pm 42$ & $123 \pm 27$ & 0,00 \\
\hline \multicolumn{4}{|c|}{1 Índice de masa corporal $\left(\mathrm{Kg} / \mathrm{m}^{2}\right)$} \\
\hline
\end{tabular}

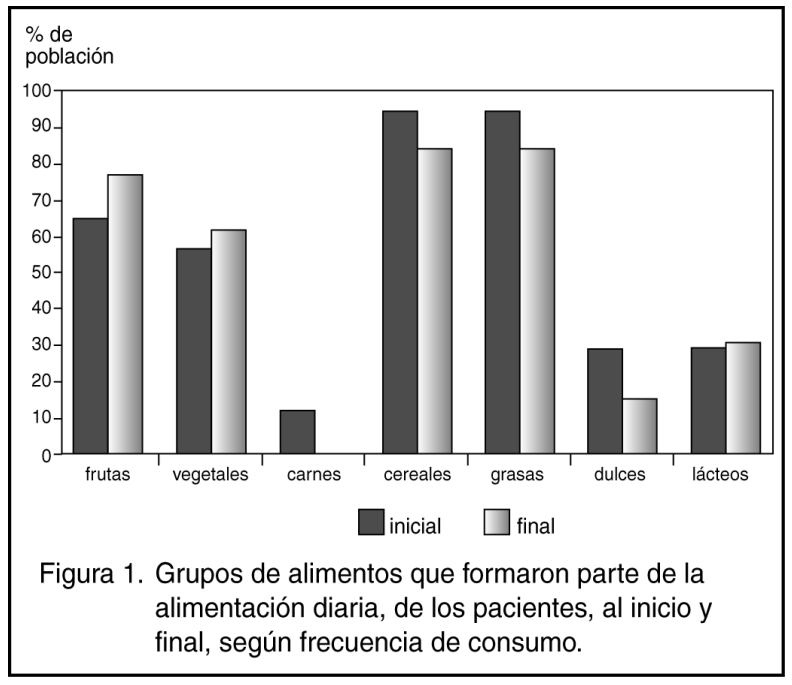

programa, en lo que respecta a los macronutrientes, se observó una disminución en el consumo de carbohidratos y un leve aumento en el consumo de grasas totales, cambios estadísticamente no significativos (cuadro 2). En la frecuencia de consumo diario, se observó que durante el programa, los pacientes disminuyeron el consumo de carnes, dulces, grasas y harinas y aumentaron el consumo de frutas, vegetales y lácteos (Figura 1).

El cuadro 3 presenta la puntuación media total de calidad de vida al inicio y al final del estudio. La puntuación media total descendió en seis puntos respecto al inicio, lo que pone de manifiesto que los pacientes percibieron una mejor calidad de vida. El análisis comparativo realizado entre la puntuación media total de CV de ambos cortes no fue estadísticamente significativo.

Uno de los resultados que se obtuvo de este programa y quizás el de mayor beneficio para los pacientes, fue la conformación de la asociación cartaginesa de pacientes cardiópatas, grupo que se reúne una vez a la semana y realiza sesiones de actividad física (ejercicios de bajo impacto) y educativas sobre temas de interés con profesionales invitados por ellos mismos.

\section{Discusión}

En la actualidad la Organización Mundial de la Salud (OMS) recomienda la inclusión de PRC dentro del marco de la prevención secundaria de accidentes cardiovasculares, con la intención de conseguir una supervivencia más larga en las mejores condiciones posibles, lo que constituye el fin primordial de la rehabilitación cardiaca ${ }^{3}$.

Los PRC han de ser siempre multifactoriales, en el sentido de que deben abarcar todas las esferas. No deben considerarse en ningún caso como un programa aislado de ejercicios físicos o entrenamiento, sino que debe incluirse dentro de la prevención secundaria que deben seguir los pacientes coronarios ${ }^{2}$.

22 AMC, enero-marzo 2003, vol 45 (1) 


\begin{tabular}{|c|c|c|}
\hline \multicolumn{3}{|c|}{$\begin{array}{c}\text { Cuadro } 2 \\
\text { Composición nutricional de la alimentación } \\
\text { de los pacientes del } \\
\text { Programa de Rehabilitación Cardiaca } \\
\text { Hospital Max Peralta, } 2000\end{array}$} \\
\hline & Inicio & Final \\
\hline Energía (Kcal) ${ }^{1}$ & $1877 \pm 783$ & $1704 \pm 634$ \\
\hline Carbohidratos $^{1}$ & 62 & 56 \\
\hline Proteína ${ }^{1}$ & 15,3 & 15,4 \\
\hline Grasas total $^{1}$ & 21,5 & 26,1 \\
\hline Grasas saturada $^{1}$ & 7,6 & 7,5 \\
\hline Grasas poliinsaturada ${ }^{1}$ & 4,4 & 5,7 \\
\hline Grasas monoinsaturada ${ }^{1}$ & 7,3 & 9,3 \\
\hline Colesterol (mg) & $203 \pm 161$ & $135 \pm 66$ \\
\hline Fibra $(g)$ & $25,6 \pm 9,8$ & $27,4 \pm 12,6$ \\
\hline
\end{tabular}

El $70 \%$ de los pacientes de este estudio presentaron factores de riesgo modificables asociadas a las enfermedades cardiovasculares, lo que nos permitió intervenir con el fin de mejorar su calidad de vida, que es el objetivo perseguido por los PRC ya que existe evidencia científica de que un adecuado control de los factores de riesgo,permite una correcta prevención secundaria en pacientes con cardiopatía isquémica, la cual se traduce en una disminución de la morbimortalidad ${ }^{11}$.

A todo paciente coronario se le deben realizan las exploraciones necesarias para conocer su riesgo futuro, y estudiar tan pronto como sea posible dentro de la fase aguda o inmediatamente después de la revascularización, su situación psicológica, social, familiar y laboral con el objetivo de mejorarla, es necesario informar y educar al paciente y a sus familiares sobre todos los aspectos referentes a su enfermedad, factores de riesgo, recuperación, readaptación laboral y reintegración familiar y social, con el fin de mejorar su calidad de vida ${ }^{7}$. En el presente estudio la integración de la familia al proceso educativo ayudó al mejoramiento de la calidad de vida no sólo del paciente sino también del familiar que lo acompañaba.

En nuestro estudio, la información suministrada por la escala utilizada para medir la calidad de vida, evidenció una mejoría que no fue significativa; sin embargo, se pudo observar un incremento en el estado de ánimo y motivación en las personas participantes. También se detectan beneficios en el perfil lipídico, sobre todo en los valores de las lipoproteínas de baja densidad, lipoproteína mayormente relacionada con el riesgo cardiovascular ${ }^{12}$.

La readecuación de la composición nutricional, así como las modificaciones en los hábitos alimentarios, relacionados con el mejoramiento en el perfil lipídico y en la reducción de peso corporal, están asociados también al incremento de la actividad física ${ }^{13,14}$.

El ejercicio físico adecuado, los consejos dietéticos, la corrección de los factores de riesgo coronario, entre los que es
Cuadro 3

Calidad de vida según áreas, al inicio y final el Programa de Rehabilitación Cardiaca

\begin{tabular}{lrr}
\hline Áreas & $\begin{array}{r}\text { Inicio } \\
\text { (puntos) }\end{array}$ & $\begin{array}{r}\text { Final } \\
\text { (puntos) }\end{array}$ \\
\hline Salud & $19,3 \pm 6,5$ & $17,7 \pm 5,4$ \\
Sueño y descanso & $6,8 \pm 3,1$ & $6,3 \pm 2,9$ \\
Comportamiento emocional & $5,7 \pm 2,7$ & $5,5 \pm 2,7$ \\
Proyectos del futuro & $5,8 \pm 2,2$ & $5,4 \pm 2,4$ \\
Movilidad & $14,3 \pm 5,9$ & $11,9 \pm 5,5$ \\
Relaciones sociales & $16,5 \pm 5,7$ & $16,4 \pm 6,2$ \\
Comportamiento de alerta & $7,1 \pm 2,4$ & $7,3 \pm 3,7$ \\
Comunicación & $5,9 \pm 2,9$ & $6,5 \pm 2,2$ \\
Tiempo y ocio & $14,5 \pm 4,1$ & $12,8 \pm 4,5$ \\
Calidad de vida global & $96,0 \pm 22,9$ & $89,7 \pm 27,3$ \\
\hline
\end{tabular}

fundamental la prohibición absoluta de fumar, y el mantenimiento de unas directrices en el comportamiento psicológico, evitando situaciones nocivas de estrés, contribuyen a crear en los enfermos un nuevo estilo, objetivo fundamental de la RC y que tiene, como consecuencia, alcanzar una mejor calidad de vida.

Se sabe que, con envejecimiento de la población aumentara el número absoluto de infartos y por ende la prevalencia general de la cardiopatía coronaria sin embargo las nuevas tecnologías y tratamientos permiten una intervención mejorada con trombolíticos y revascularización aguda, conduciendo a estancias intra hospitalarias más breves que permitirán a más enfermos participar en programas de rehabilitación por lo que la rehabilitación cardiaca y áreas relacionadas quizás estarán recibiendo más atención en el futuro cercano.

\section{Conclusión}

Se concluye, que la educación es el elemento primordial para establecer este tipo programas en un sistema de salud cuyo objetivo es la curación, sin embargo, la implementación del mismo se hace difícil, por el desconocimiento sobre la importancia y beneficios de los PRC, falta de interés de las autoridades de salud por este tipo de actividades, falta de contenido presupuestario, personal especializado, espacio físico y motivación del personal de salud para realizar actividades de promoción y prevención de salud.

\section{Agradecimientos}

Agradecemos al Dr. Agustín Solís, Cardiólogo, a la Dra. Xinia Monestel, fisioterapia, a la Licda. Rosella Riggioni del Departamento de Nutrición, a la Técnica en Fisioterapia Rosa Elena Sánchez Calvo y al departamento de Microbiología, quienes colaboraron desinteresadamente durante la realización de este programa. 


\section{Abstract}

Implementation of Cardiac Rehabilitation Program (Phase II) in the Max Peralta Hospital

Justification and objective: A cardiac rehabilitation program (CRP) plays a very important role in the secondary prevention of cardiovascular diseases. The results obtained by the first group patients of that participated in a CRP developed at the Max Peralta Hospital in Cartago are presented in this article.

Methods: A CRP was implemented for patients who had suffered a cardiac event the program, consisted of group educational sessions, held once a week for three hours during three months. The lipid profile, anthropometrics, diet and quality of life were evaluated at the beginning and at the end of the study period.

Results: 15 patients participated, the average age was 59 years, more than $70 \%$ of them presented risk factors associated with cardiovascular diseases, $59 \%$ were overweight or were frankly obese (BMI >25). At the end the lipid profile had improved, mainly cholesterol and LDL levels. They were eating a diet nutritionally balanced, and the frequency of consumption of fruits, vegetables, diary products had increased. On the contrary, the frequency of intake of meats, candies, fats and cereals diminished. Quality Life improved from the beginning $(96 \pm 22,9)$ until the end $(89,7 \pm 27,3)$.

Conclusion: Even though the establishment of CRP in hospitals is difficult, a clear improvement of the patients physical and emotional aspects was observed.

Key words: Cardiac rehabilitation program. Lipids. Education. Nutrition. Diet. Quality of Life. Cardiac event.

\section{Referencias}

1. Ministerio de Salud. Memoria Anual 1999 (San José, Costa Rica): Ministerio de Salud 2000.

2. Velasco J, Maroto J. Rehabilitación cardiaca. Evolución histórica y situación actual. Rev Esp Cardiol. 1995; 48 (Supl 1):1-7.

3. OMS. Rehabilitación después de las enfermedades cardiovasculares, en especial atención a los países en desarrollo. 1993. Serie de informes técnicos 831 .

4. Pashkow F. Issues in contemporary cardiac rehabilitation; a historical perspective J Am Coll Cardiol. 1993; 21(3):822-834.

5. Hedback B, Perk J, Wodlin P. Long-term reduction of cardiac mortality after myocardial infarction:10-year results of a comprehensive rehabilitation programme. Eur Heart J. 1993; 14(6):831-835.

6. Lavie C, Milani R. Benefits of cardiac rehabilitation and exercise training in elderly women. Am J Cardiol. 1997; 79(5): 664-666.

7. Jiménez M, Gómez C, Sáenz C, Del -campo S, Lacasa J, Pombo M. Calidad de vida y apoyo social en pacientes con infarto agudo de miocardio no complicado. Rev Esp Cardiol. 1999; 52 : 467-474.

8. Tormo V,1995. Rehabilitación cardiaca a largo plazo. Rev Esp Cardiol. 48 (supl 1)

9. Sosa V, Ubiera J, Martínez E,Cantapiedra J, García M,Delcán J. 1995. Protocolos de rehabilitación hospitalaria en fase I y Fase II. Rev Esp Cardiol. 48 (supl 1)

10. Velasco JA, del Barrio V, Mestre MV, Penas C, Ridocci F. Validación de un nue vo cuestionario para evaluar la calidad de vida en pacientes postinfarto. Rev Esp Cardiol. 1993; 46 : 552-558.

11. Artigao R. Control de factores de riesgo en los programas de rehabilitación cardiaca. Rev Esp Cardiol. 1995; 48 (Supl 1):13-21.

12. Castelli W. Epidemiology of coronary disease: The Framingham Study. Am J Med. 1994; 27:4-11.

13. Programa nacional de educación sobre el colesterol. Resumen del II informe del Panel de Expertos sobre Detección, evaluación y tratamiento de la hipercolesterolemia en adultos. JAMA 1993; 269(23): 3015-3023.

14. Friedman D, Williams A, Levine B. Compliance and efficacy of cardiac rehabilitation and risk factor modification in the medically indigent. Am J Clin Nutr. 1997; 79(3):281-5.

\section{AMC, enero-marzo 2003, vol 45 (1)}

